\title{
The impact of participatory budgeting on health and wellbeing: a scoping review of evaluations
}

\author{
Mhairi Campbell ${ }^{1 *}$ (D), Oliver Escobar ${ }^{2,3}$, Candida Fenton $^{3}$ and Peter Craig ${ }^{1,2}$
}

\begin{abstract}
Background: Participatory budgeting (PB), citizens deliberating among themselves and with officials to decide how to allocate funds for public goods, has been increasingly implemented across Europe and worldwide. While PB is recommended as good practice by the World Bank and the United Nations, with potential to improve health and wellbeing, it is unclear what evaluations have been conducted on the impact of PB on health and wellbeing.

Methods: For this scoping review, we searched 21 databases with no restrictions on publication date or language. The search term 'participatory budget' was used as the relevant global label for the intervention of interest. Studies were included if they reported original analysis of health, social, political, or economic and budgetary outcomes of PB. We examined the study design, analysis, outcomes and location of included articles. Findings are reported narratively.

Results: From 1458 identified references, 37 studies were included. The majority of evaluations $(n=24)$ were of PB in South America, seven were in Europe. Most evaluations were case studies $(n=23)$ conducting ethnography and surveys, focussing on political outcomes such as participation in PB or impacts on political activities. All of the quantitative observational studies analysing population level data, except one in Russia, were conducted in South America.

Conclusion: Despite increasing interest in $\mathrm{PB}$, evaluations applying robust methods to analyse health and wellbeing outcomes are scarce, particularly beyond Brazil. Therefore, implementation of PB schemes should be accompanied by rigorous qualitative and quantitative evaluation to identify impacts and the processes by which they are realised.
\end{abstract}

\section{Background}

Participatory budgeting (PB) aims to democratically allocate public money for local services, enabling communities to decide how public funds are spent [1]. It entails a multi-stage process, which typically concludes with a vote, where citizens deliberate among themselves and with government officials to allocate funds for public goods [2]. Since it's origination in 1989 in Porto Alegre, Brazil, PB has been implemented in many countries across North and South America, Europe and Asia, including many high income countries [3, 4]. International institutions including the World Bank, the Organisation

\footnotetext{
* Correspondence: Mhairi.Campbell@glasgow.ac.uk

${ }^{1}$ MRC/CSO Social and Public Health Sciences Unit, University of Glasgow, 200

Renfield Street, Glasgow G2 3QB, UK

Full list of author information is available at the end of the article
}

for Economic Cooperation and Development (OECD), the United Nations and the UK Department for International Development recognise PB as good practice $[3,5,6]$ and worldwide $\mathrm{PB}$ schemes distribute multimillion dollars/ euros [7]. In Porto Alegre, $\mathrm{PB}$ has been reported to increase public spending in the poorest areas of the city, reduce administrative costs and improve citizen participation [8].

Literature on $\mathrm{PB}$ abounds, but to our knowledge, there has been no systematic assessment of the impact of $\mathrm{PB}$ on health and wellbeing across the world. PB may influence health and wellbeing via several pathways: increase in democratic participation; greater community cohesion; improved allocation of funding to public services prioritised by the community; and increased access to medical services via increased spending on healthcare 
and social determinants of health such as sanitation or housing. Table 1 outlines the possible stages of how PB can influence health and wellbeing, informed by key literature $[3,6,9,10]$.

In order to establish the strength and reach of the existing evidence base for $\mathrm{PB}$ as a way of improving democratic participation, community cohesion, delivery of public services, and population health outcomes, we conducted a systematic scoping review of international literature to identify evaluations of PB schemes. Specifically, we examined what methods have been used to evaluate $\mathrm{PB}$ processes, what outcomes have been used to investigate the effects of $\mathrm{PB}$, in which countries and at what geographic scale (e.g. neighbourhood, municipality, region). This scoping review is timely as there is growing interest from governments across Europe and beyond, in $\mathrm{PB}$ as a process for allocating public funds with the potential to address inequalities and increase community empowerment and cohesion [11, 12]. As PB is adapted and adopted in countries beyond Brazil, there can be less focus on social reform that was integral to the original process [13], with implications for benefits to health and wellbeing.

\section{Methods}

The methodology for this review was guided by recent recommendations for conducting scoping reviews $[14,15]$, the protocol is available [16].

\section{Inclusion criteria}

The review included studies of adults and children, as individuals or groups in communities involved in, or impacted by, PB. The intervention of interest was $\mathrm{PB}$ defined as communities deciding collectively how public funds are allocated. We did not include any form of individual budgetary decision-making (e.g. processes where individuals have personal management of welfare

Table 1 How PB can affect the health, social, democratic and economic outcomes of individuals The intervention, participatory budgeting, is expected to impact on the
health, social and economic outcomes of individuals involved through the
following stages, derived from UN-Habitat and World Bank reports
(Cabannes 2004, Shah 2007) and (Boulding and Wampler 2010, page 126):
- Participation: communities can decide how designated public money is
spent.

- Collaboration: being involved in the PB decision process enables citizens to exercise political rights, develop civic skills and build social cohesion.

- Prioritisation: improvements in priority public services may improve the wellbeing of individuals in that community, either directly through impacts on their health (e.g. reduction in disease, better access to medical services) or via social determinants of health (e.g. housing, education).

- Allocation: distribution of resources according to identified needs results in greater efficiency in the allocation of public funds, and greater accountability of budgetary procedures. budgets, or employees have the opportunity to participate in defining their budget). Areas of interest included political, economic and budgetary, and health and social outcomes occurring at individual, population and systems levels. Political outcomes included outcomes at individual level such as participation, and systems level outcomes such as the democratic process at a local level. Economic and budgetary outcomes included outcomes at population level such as health or social service provision, housing, patterns of spending as the mechanism for impact on other outcomes, and provision of public goods (e.g. public parks, public safety). Health and social outcomes included outcomes at individual level such as health, wellbeing, self-efficacy, and empowerment, and at population level such as measurements of inequalities (e.g. poverty rates). We included any type of study design, quantitative, qualitative or mixed methods, with and without control groups or comparisons, which used primary analysis and reported on an evaluation of the $\mathrm{PB}$ system in relation to any of the outcomes listed above. This included peer reviewed published articles, books, reports and grey literature such as conference papers or working papers. There was no limitation on publication language or date; we did not find publications on PB prior to the 1980s when the process was established.

\section{Literature search}

Twenty-one electronic databases were searched in October 2016, with an updated search conducted in May 2018. The databases included peer reviewed articles and grey literature, and aimed to cover a broad range of health, social, political and economic literature, see Additional file 1: Table S1 for full list. No filters or terms were used to capture evaluation studies as evaluation studies are not indexed as such in bibliographic databases. No language or date limits were applied to the literature searches. Further articles from the review authors' collections were also included. The search term 'participatory budget' was used as this is the relevant global label used for the process that we wanted to examine; the term is used internationally to identify this specific process. The search results were first screened by title and abstract, with $10 \%$ independently screened by two reviewers. The full text was then screened, with duplicate screening of $10 \%$, and disagreements resolved by discussion and in consultation with the third reviewer.

\section{Data extraction and collation}

A data extraction template was developed in Microsoft Excel, tested and agreed by the review team. Duplicate data extraction was conducted on $20 \%$ of the included studies, see Additional file 1: Table S2 for data extraction template. As this was a scoping review, aiming to map 
the amount and type of evidence available on PB in relation to impacts on health and wellbeing, the literature was not formally appraised for methodological quality. The data were collated in summary tables and the results reported narratively.

\section{Results}

The literature search identified 1458 citations. After de-duplication and screening, 39 articles reporting on 37 studies met the inclusion criteria, see Fig. 1 for a flow diagram of the screening process. A summary of characteristics table provides details of the included studies' study design, country, data sources, methods of analysis, outcomes of interest, and source of funding (Table 2), and Additional file 1: Table S3 provides further details of the studies. Most of the studies that met the inclusion criteria were published in English; three of the included texts were in Portuguese and one in Spanish. Several evaluations had been published in English and the language of the country of origin.
In which countries and policy sectors have PB processes been evaluated, and at what geographic scale?

The majority of the studies report on evaluations of $\mathrm{PB}$ in South America $(n=24)$, most in Brazil $(n=19)$, many $(n=7)$ focussed on Porto Alegre, where PB originated. The remaining South American studies were located in Peru $(n=3)$, Colombia $(n=1)$, and one multi-national study set in Brazil, Ecuador and Venezuela. There were seven evaluations of PB in Europe. One evaluation was conducted in the United States; three were set in Asia; and one study of PB was conducted in Africa. There was also one article collating case studies examining the impact of PB across twenty cities worldwide: South America $(n=12)$; Africa $(n=4)$; Europe $(n=2)$; Asia $(n=1)$; and the United States $(n=1)$.

The PB schemes operated and were evaluated at municipal level in $89 \%(n=33)$ of the studies. The remaining four studies evaluated PB schemes at neighbourhood level [17-20]. The source of funding for the evaluation was reported in 59\% of the studies; $38 \%$ government funding or non-government organisation, $21 \%$ reported

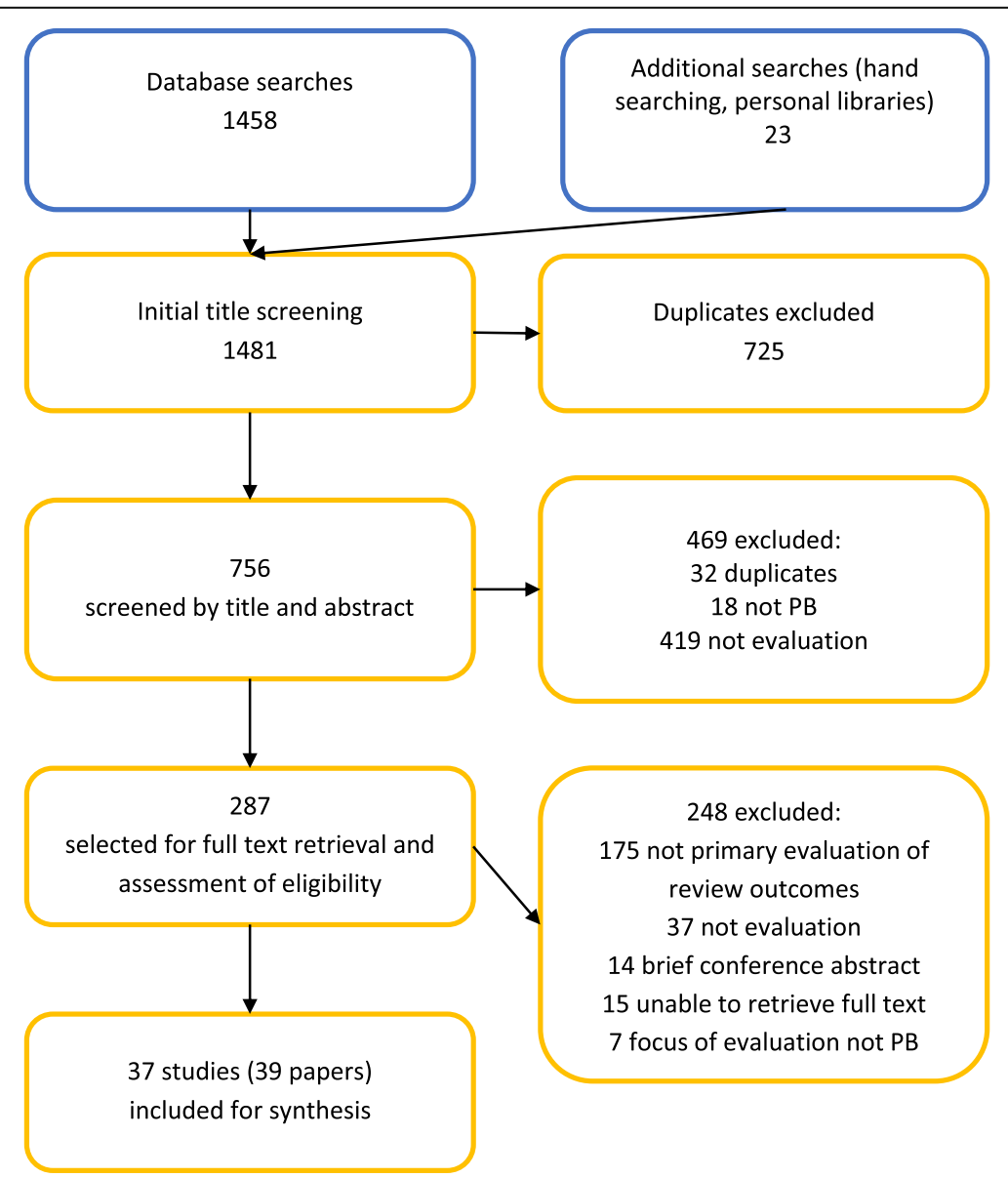

Fig. 1 Literature screening flow diagram 
Table 2 Included studies: study design, country, data sources, methods of analysis, and outcomes of interest

\begin{tabular}{|c|c|c|c|c|c|}
\hline $\begin{array}{l}\text { First author } \\
\text { date }\end{array}$ & Country & $\begin{array}{l}\text { Data source(s) Individual/ } \\
\text { aggregate data }\end{array}$ & Analysis & Outcome(s) & $\begin{array}{l}\text { Source of funding for } \\
\text { evaluation } \\
\text { Investigator connection }\end{array}$ \\
\hline \multicolumn{6}{|c|}{ Randomised controlled trials } \\
\hline $\begin{array}{l}\text { Beuermann } \\
2014 \text { [21] }\end{array}$ & Russia & $\begin{array}{l}\text { Municipal data survey }(n=109) \\
\text { Household survey }(n=1645) \\
\text { political representatives }(n=109) \\
\text { Aggregate + Individual }\end{array}$ & $\begin{array}{l}\text { Fixed effects } \\
\text { regression } \\
\text { comparing } 2 \text { PB } \\
\text { treatment areas and } \\
\text { control non-PB areas }\end{array}$ & $\begin{array}{l}\text { Tax revenue. Allocation of PB } \\
\text { funds. Participation in PB. }\end{array}$ & $\begin{array}{l}\text { World Bank (IDB); } \\
\text { Government of the } \\
\text { Russian Federation. } \\
\text { PB consultants for the } \\
\text { RCT conducted } \\
\text { evaluation }\end{array}$ \\
\hline \multicolumn{6}{|c|}{ Quantitative observational studies } \\
\hline $\begin{array}{l}\text { Schneider } \\
2002[22]\end{array}$ & Brazil & $\begin{array}{l}\text { Municipal data }(n=497) \\
\text { Aggregate }\end{array}$ & $\begin{array}{l}\text { Linear regression } \\
\text { comparing } \mathrm{PB} \text { and } \\
\text { non-PB } \\
\text { municipalities }\end{array}$ & $\begin{array}{l}\text { Impact on participation in } \mathrm{PB} \\
\text { by spending of } \mathrm{PB}\end{array}$ & $\begin{array}{l}\text { None stated } \\
\text { Academic investigator }\end{array}$ \\
\hline $\begin{array}{l}\text { Biderman } \\
2007[24]\end{array}$ & Brazil & $\begin{array}{l}\text { RAIS administrative records, } \\
\text { census data } \\
\text { Aggregate }\end{array}$ & $\begin{array}{l}\text { Fixed effects } \\
\text { regression } \\
\text { comparing PB and } \\
\text { non-PB } \\
\text { municipalities }\end{array}$ & $\begin{array}{l}\text { Tax revenue, infant mortality, } \\
\text { education }\end{array}$ & $\begin{array}{l}\text { Part funded by World } \\
\text { Bank } \\
\text { Academic investigators }\end{array}$ \\
\hline $\begin{array}{l}\text { Wampler } \\
\text { 2007/2012 [30, } \\
\text { 31] }\end{array}$ & Brazil & $\begin{array}{l}\text { Survey }(n=833) \\
\text { Individual }\end{array}$ & Logistic regression & $\begin{array}{l}\text { Impact on further political } \\
\text { activities }\end{array}$ & $\begin{array}{l}\text { National Science } \\
\text { Foundation } \\
\text { Academic investigator }\end{array}$ \\
\hline $\begin{array}{l}\text { World Bank } \\
2008[8]\end{array}$ & Brazil & $\begin{array}{l}\text { Municipal data }(n=150+) \\
\text { Survey }(n=1300) \\
\text { Aggregate }+ \text { Individual }\end{array}$ & $\begin{array}{l}\text { Difference in } \\
\text { differences } \\
\text { comparing PB and } \\
\text { non-PB } \\
\text { municipalities }\end{array}$ & $\begin{array}{l}\text { Municipal budget PB } \\
\text { expenditure, poverty, } \\
\text { sanitation, infant mortality, } \\
\text { education, participation of } \\
\text { disadvantaged groups }\end{array}$ & $\begin{array}{l}\text { Social Development Unit } \\
\text { of the Latin America and } \\
\text { Caribbean Region } \\
\text { (LCSSO) and the Social } \\
\text { Development } \\
\text { Department (SDV) of the } \\
\text { World Bank. } \\
\text { NGO investigation }\end{array}$ \\
\hline
\end{tabular}

\begin{tabular}{|c|c|c|c|c|c|}
\hline $\begin{array}{l}\text { Boulding } \\
2010[9]\end{array}$ & Brazil & $\begin{array}{l}\text { Brazilian Institute of Geography } \\
\text { and Economics, census } \\
\text { Aggregate }\end{array}$ & $\begin{array}{l}\text { Linear regression } \\
\text { comparing } \mathrm{PB} \text { and } \\
\text { non-PB } \\
\text { municipalities }\end{array}$ & $\begin{array}{l}\text { Poverty, inequality (GINI), life } \\
\text { expectancy, infant mortality, } \\
\text { adult and child literacy. } \\
\text { Allocation of PB funding }\end{array}$ & $\begin{array}{l}\text { None stated. Academic } \\
\text { evaluation }\end{array}$ \\
\hline $\begin{array}{l}\text { Garcia } \\
2011[29]\end{array}$ & Spain & $\begin{array}{l}\text { Center for Sociological Research } \\
\text { Survey }(n=1004) \\
\text { Individual }\end{array}$ & $\begin{array}{l}\text { Linear regression } \\
\text { analysis } \\
\text { comparing district } \\
\text { council system }\end{array}$ & $\begin{array}{l}\text { Participation of women, impact } \\
\text { on further political activities }\end{array}$ & $\begin{array}{l}\text { Women's Institute and } \\
\text { the National R \& D \& I } \\
\text { Plan of the Government } \\
\text { of Spain. } \\
\text { Academic investigator }\end{array}$ \\
\hline $\begin{array}{l}\text { Borba } \\
2012[32]\end{array}$ & Brazil & $\begin{array}{l}\text { NUPESAL (Nucleo de Pesquisas } \\
\text { Sobre a America Latina) } \\
\text { Survey }(n=533) \\
\text { Individual }\end{array}$ & $\begin{array}{l}\text { Logistic regression } \\
\text { analysis comparing } \\
\text { residents involved in } \\
\mathrm{PB} \text { and non-PB in- } \\
\text { volved residents }\end{array}$ & $\begin{array}{l}\text { Impact on further political } \\
\text { activities, participation in } \mathrm{PB}\end{array}$ & $\begin{array}{l}\text { None stated } \\
\text { Academic investigator }\end{array}$ \\
\hline $\begin{array}{l}\text { Jaramillo } \\
2013[23]\end{array}$ & Peru & $\begin{array}{l}\text { Municipal data }(n=219) \text { from } \\
\text { MSUNASS, PB-DNPP, MEF-SIAF, } \\
\text { ONPE, JNE, RENAMU. } \\
\text { Interviews ( } n=\text { ?) in } 4 \text { PB areas } \\
\text { Aggregate + Individual }\end{array}$ & $\begin{array}{l}\text { Linear regression } \\
\text { analysis comparing } \\
2007 \text { data with } 2001\end{array}$ & $\begin{array}{l}\text { Sanitation (water coverage and } \\
\text { continuity) }\end{array}$ & $\begin{array}{l}\text { Institutional Capacity } \\
\text { Strengthening Fund } \\
\text { (ICSF), managed by Inter- } \\
\text { American } \\
\text { Development Bank (IDB), } \\
\text { Government of the } \\
\text { People's Republic of } \\
\text { China. } \\
\text { NGO investigation }\end{array}$ \\
\hline $\begin{array}{l}\text { Da Silva } \\
2014[33]\end{array}$ & Brazil & $\begin{array}{l}\text { Brazilian Institute of Geography } \\
\text { and Statistics } \\
\text { Aggregate }\end{array}$ & $\begin{array}{l}\text { Cross tabulation } \\
\text { descriptive analysis }\end{array}$ & $\begin{array}{l}\text { PB investment per capita by } \\
\text { deprived area. Impact on PB } \\
\text { results by type of PB }\end{array}$ & $\begin{array}{l}\text { Not stated } \\
\text { Academic investigator }\end{array}$ \\
\hline $\begin{array}{l}\text { Goncalves } \\
2014[25]\end{array}$ & Brazil & $\begin{array}{l}\text { Municipal data ( } n=3651 \text { ) from } \\
\text { Brazilian Institute of Geography } \\
\text { and Economics, census } \\
\text { Aggregate }\end{array}$ & $\begin{array}{l}\text { Fixed effects } \\
\text { regression } \\
\text { comparing PB and } \\
\text { non-PB } \\
\text { municipalities }\end{array}$ & $\begin{array}{l}\text { Municipal budget PB } \\
\text { expenditure on education, } \\
\text { housing, sanitation, cultural. } \\
\text { Poverty, infant mortality }\end{array}$ & $\begin{array}{l}\text { Not stated } \\
\text { Academic investigator }\end{array}$ \\
\hline
\end{tabular}


Table 2 Included studies: study design, country, data sources, methods of analysis, and outcomes of interest (Continued)

\begin{tabular}{|c|c|c|c|c|}
\hline $\begin{array}{l}\text { First author } \\
\text { date }\end{array}$ & Country & $\begin{array}{l}\text { Data source(s) Individual/ } \\
\text { aggregate data }\end{array}$ & Analysis & Outcome(s) \\
\hline $\begin{array}{l}\text { Touchton } \\
2014 \text { [26] }\end{array}$ & Brazil & $\begin{array}{l}\text { Municipal data }(n=253) \text { from } \\
\text { Brazilian Institute of Geography } \\
\text { and Economics, census } \\
\text { Aggregate }\end{array}$ & $\begin{array}{l}\text { Random effects } \\
\text { regression } \\
\text { comparing PB and } \\
\text { non-PB } \\
\text { municipalities }\end{array}$ & $\begin{array}{l}\text { Municipal budget PB } \\
\text { expenditure on healthcare and } \\
\text { sanitation. Infant mortality }\end{array}$ \\
\hline $\begin{array}{l}\text { Jaramillo } \\
2015[27]\end{array}$ & Peru & $\begin{array}{l}\text { Municipal data } \\
\text { Survey } 2 \text { wave }(n=100) \\
\text { Aggregate }+ \text { Individual }\end{array}$ & $\begin{array}{l}\text { Linear regression } \\
\text { analysis comparing } \\
2010 \text { data with } 2007\end{array}$ & $\begin{array}{l}\text { Number and quality of } \\
\text { agricultural services }\end{array}$ \\
\hline
\end{tabular}

Source of funding for

evaluation

Investigator connection

Boise State University's

College of Social Science and Public Affairs

Academic investigators

National Science

Foundation and the

Boren National Security

Education Program

Not for Profit research

centre/academic

investigators

Grillos $2017 \quad$ Indonesia Municipal data Aggregate

[28]

Single case studies

$\begin{array}{ll}\text { Abers } & \text { Brazil } \\ 1998[34] & \\ \text { Baiocchi } & \text { Brazil } \\ 2001[16] & \end{array}$

2001 [16]

razil

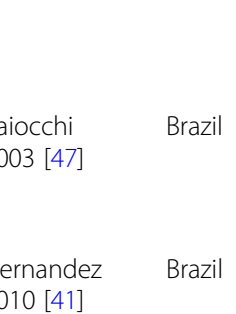

2010 [41] $\quad \begin{aligned} & \text { Observation at PB assemblies. } \\ & \text { Data from Data from } \\ & \text { Coordenadoria do Orçamento } \\ & \text { Participativo (COP) } \\ & \text { Individual }\end{aligned}$

\begin{tabular}{|c|c|c|}
\hline $\begin{array}{l}\text { Walker } \\
\text { 2013/2016 [44, } \\
45]\end{array}$ & Brazil & $\begin{array}{l}\text { Interviews ( } n=20) \text {, participant } \\
\text { observation in study area } \\
\text { Individual }\end{array}$ \\
\hline
\end{tabular}

$\begin{array}{llll}\begin{array}{l}\text { Stewart } \\ \text { 2014 [20] }\end{array} & \text { USA } & \begin{array}{l}\text { Municipal data (City of } \\ \text { Chicago's Aldermanic Menu } \\ \text { reports), census data, PB } \\ \text { evaluation reports } \\ \text { Aggregate + Individual }\end{array} & \begin{array}{l}\text { Qualitative + } \\ \text { descriptive } \\ \text { quantitative }\end{array} \\ & & \begin{array}{l}\text { Interviews }(n=18), \text { survey }(n= \\ \text { Célérier }\end{array} \quad \text { Brazil } & \text { 46), participant observation }\end{array}$

\begin{tabular}{|c|c|c|}
\hline $\begin{array}{l}\text { Kendall } \\
2015 \text { [18] }\end{array}$ & Malawi & Survey, data collection (5 sites) \\
\hline
\end{tabular}

McNulty $\quad$ Peru Interviews $(\mathrm{n}=$ unclear), $\quad$ Single case study

Linear regression

city sub-districts

Qualitative + descriptive quantitative

Qualitative + descriptive quantitative descriptive

Interviews ( $n=65)$, survey $(n=$ 74), participant
PB assemblies Individual observation in study area Individual analysis comparing

Allocation of PB funding

Harvard Kennedy School Indonesia Program Academic investigator

Mobilisation of neighbourhood Not stated residents. Impact on further political activities. Participation in PB by low income.

Participation in PB of
disadvantaged groups (women,
low income, low education).
low income, low education).

National Science

Foundation, Inter American Foundation, and University of Wisconsin Academic investigator

Use of PB assembly meetings for further community activities.

Participation in PB of disadvantaged groups: AfroBrazilians, senior citizens, children and adolescents, the GLBT community, women, indigenous groups, homeless, and people with disabilities.

Political and social learning (negotiations for housing)

Participation in PB. Allocation of $\mathrm{PB}$ funds.

Not stated Academic investigator

Tinker Foundation, the Center for Latin American and Caribbean Studies and the Graduate School at Brown University Academic investigator

National Science Foundation and the Foundation for Urban and Regional studentship Academic investigators

None stated Academic investigators

Participation in PB. Impact on political activities.

Impact on democratic processes relating to the school, local communities and funding bodies. government data
Participation in PB by women.
HEC Foundation and of the French Ministry of Foreign Affairs

TAG Philanthropic

Foundation Academic investigators

Franklin and Marshall College and The American Association of University Women Academic investigator Academic investigators 
Table 2 Included studies: study design, country, data sources, methods of analysis, and outcomes of interest (Continued)

\begin{tabular}{|c|c|c|c|c|c|}
\hline $\begin{array}{l}\text { First author } \\
\text { date }\end{array}$ & Country & $\begin{array}{l}\text { Data source(s) Individual/ } \\
\text { aggregate data }\end{array}$ & Analysis & Outcome(s) & $\begin{array}{l}\text { Source of funding for } \\
\text { evaluation } \\
\text { Investigator connection }\end{array}$ \\
\hline $\begin{array}{l}\text { Hajdarowicz } \\
2018[63]\end{array}$ & Columbia & $\begin{array}{l}\text { Interviews }(n=19) \text {, participant } \\
\text { observation }\end{array}$ & Qualitative & Participation in PB by women. & $\begin{array}{l}\text { None stated } \\
\text { Academic investigator }\end{array}$ \\
\hline \multicolumn{6}{|c|}{ Multiple case studies } \\
\hline $\begin{array}{l}\text { Nylen } \\
2003 \text { [37] }\end{array}$ & & $\begin{array}{l}\text { Interviews ( } \mathrm{n}=\text { unclear), survey } \\
(n=1280) \\
\text { Individual }\end{array}$ & $\begin{array}{l}\text { Qualitative + } \\
\text { descriptive } \\
\text { quantitative }\end{array}$ & $\begin{array}{l}\text { Participation in PB of } \\
\text { disadvantaged groups (women, } \\
\text { low education). Empowerment, } \\
\text { impact on further political } \\
\text { activities. }\end{array}$ & Academic investigator \\
\hline $\begin{array}{l}\text { Cabannes } \\
2005[49]\end{array}$ & $\begin{array}{l}\text { South } \\
\text { America } \\
\text { (multiple) }\end{array}$ & $\begin{array}{l}\text { Survey ( } \mathrm{n}=4 \text { PB schemes) } \\
\text { ? }\end{array}$ & $\begin{array}{l}\text { Qualitative + } \\
\text { descriptive } \\
\text { quantitative }\end{array}$ & Allocation of PB funds. & $\begin{array}{l}\text { UNDP/Habitat } \\
\text { NGO evaluation }\end{array}$ \\
\hline $\begin{array}{l}\text { Renno } \\
2010[51]\end{array}$ & Brazil & Survey 3 wave ( $n=$ unclear) & Qualitative & $\begin{array}{l}\text { Political learning. Impact on } \\
\text { political activities. }\end{array}$ & $\begin{array}{l}\text { None stated } \\
\text { Academic investigator }\end{array}$ \\
\hline $\begin{array}{l}\text { SQW } \\
\text { Consulting } \\
2011[19]\end{array}$ & UK & $\begin{array}{l}\text { Survey ( } \mathrm{n}=\text { unclear). Municipal } \\
\text { data [check] }\end{array}$ & $\begin{array}{l}\text { Qualitative + } \\
\text { descriptive } \\
\text { quantitative }\end{array}$ & Political and social learning & $\begin{array}{l}\text { Department for } \\
\text { Communities and Local } \\
\text { Government } \\
\text { Government evaluation }\end{array}$ \\
\hline $\begin{array}{l}\text { Wu } \\
2011[46]\end{array}$ & China & $\begin{array}{l}\text { Interviews ( } n=15) \text {, Survey ( } n= \\
547)\end{array}$ & Qualitative & Political and social learning & $\begin{array}{l}\text { China Development } \\
\text { Research Foundation } \\
\text { (CDRF) Chinese central } \\
\text { government, People's } \\
\text { Bank of China } \\
\text { Academic investigators }\end{array}$ \\
\hline $\begin{array}{l}\text { Bassoli } \\
2012[48]\end{array}$ & Italy & $\begin{array}{l}\text { Survey } 3 \text { wave }(2002 / 3 n=12 \text {; } \\
2005 n=4 ; 2007 / 9 n=9)\end{array}$ & Qualitative & $\begin{array}{l}\text { Democratic characteristics of } \\
\text { PB schemes: inclusion, } \\
\text { participation, the role of the } \\
\text { opposition, and transparency. }\end{array}$ & $\begin{array}{l}\text { None stated } \\
\text { Academic investigator }\end{array}$ \\
\hline $\begin{array}{l}\text { Luchmann } \\
2012[42]\end{array}$ & Brazil & $\begin{array}{l}\text { Focus group PB delegates and } \\
\text { councillors, survey }(n=47)\end{array}$ & Qualitative & Political and social learning & $\begin{array}{l}\text { CNPq (Conselho Nacional } \\
\text { de Pesquisa / National } \\
\text { Research Council) } \\
\text { Academic investigator }\end{array}$ \\
\hline $\begin{array}{l}\text { Talpin } \\
2012[38]\end{array}$ & Italy & $\begin{array}{l}\text { Interviews ( } n=12) \text {, participant } \\
\text { observation at } 54 \text { PB meetings }\end{array}$ & Qualitative & $\begin{array}{l}\text { Participation in PB. Political } \\
\text { learning. Impact on further } \\
\text { political activities. }\end{array}$ & $\begin{array}{l}\text { None stated } \\
\text { Academic investigator }\end{array}$ \\
\hline $\begin{array}{l}\text { Cabannes } \\
2015[39]\end{array}$ & $\begin{array}{l}\text { Multiple } \\
\text { across South } \\
\text { America, } \\
\text { North } \\
\text { America, } \\
\text { Africa, Asia, } \\
\text { Europe }\end{array}$ & $\begin{array}{l}\text { Interviews }(n=12) \text {, Survey } \\
(n=20) \\
\text { Aggregate }+ \text { Individual }\end{array}$ & $\begin{array}{l}\text { Qualitative + } \\
\text { descriptive } \\
\text { quantitative }\end{array}$ & $\begin{array}{l}\text { Impact on political processes of } \\
\text { municipality. Water supply, } \\
\text { sanitation, public transport, } \\
\text { roads, electricity supply }\end{array}$ & $\begin{array}{l}\text { Aid UK, UK Government } \\
\text { NGO (IIED) evaluation }\end{array}$ \\
\hline $\begin{array}{l}\text { del Prado } \\
2015 \text { [40] }\end{array}$ & Philippines & $\begin{array}{l}\text { Interviews ( } n=\text { unclear), focus } \\
\text { groups ( } n=\text { unclear). Municipal } \\
\text { data (sources unclear) } \\
\text { Individual }\end{array}$ & $\begin{array}{l}\text { Qualitative + } \\
\text { descriptive } \\
\text { quantitative }\end{array}$ & Allocation of PB funds & $\begin{array}{l}\text { Government think tank } \\
\text { evaluation }\end{array}$ \\
\hline $\begin{array}{l}\text { Džinic } 2016 \\
{[17]}\end{array}$ & $\begin{array}{l}\text { Eastern } \\
\text { Europe } \\
\text { Multiple }\end{array}$ & $\begin{array}{l}\text { Municipal data from } \\
\text { municipality websites, press and } \\
\text { official reports } \\
\text { Aggregate }\end{array}$ & $\begin{array}{l}\text { Qualitative + } \\
\text { descriptive } \\
\text { quantitative }\end{array}$ & Allocation of PB funds. & $\begin{array}{l}\text { None stated } \\
\text { Academic investigators }\end{array}$ \\
\hline $\begin{array}{l}\text { Montambeault } \\
2016[50]\end{array}$ & Brazil & $\begin{array}{l}\text { Survey }(2009 n=967,2014 \\
n=473) \\
\text { Individual }\end{array}$ & Qualitative & Participation in PB. & $\begin{array}{l}\text { Emerging Scholar grant } \\
\text { from the Fonds de la } \\
\text { Recherche du } \\
\text { Québec-Société et } \\
\text { Culture } \\
\text { Academic investigator }\end{array}$ \\
\hline $\begin{array}{l}\text { Gregorčič } \\
2016[52]\end{array}$ & $\begin{array}{l}\text { Solvenia, } \\
\text { Iceland }\end{array}$ & $\begin{array}{l}\text { Interviews ( } n=12 \text {, Solvenia), } \\
\text { participant observation (Iceland) }\end{array}$ & Qualitative & Political and social learning & $\begin{array}{l}\text { Not stated } \\
\text { Academic investigator }\end{array}$ \\
\hline
\end{tabular}


an academic funding source. Academic researchers conducted $76 \%$ of the studies; the remaining $24 \%$ were non-government organisation evaluations.

What methods have been used to evaluate PB processes? The included studies were split broadly by study methodology and the level of data used. The first category, randomised controlled trials and quantitative observational studies, lists studies that used modelling to identify the effect of the outcomes or analysed large-scale population level data (Table 2). The second category collates case studies that conducted primary data collection in the form of surveys, interviews and participant observation; some of these studies included descriptive analysis of municipal data. Almost half of the case studies $(n=10)$ used mixed method study designs (qualitative interviews or surveys and descriptive quantitative analysis). One of the observational modelling studies included qualitative methods [8].

\section{Randomised controlled trials and quantitative observational modelling studies}

We found one randomised controlled trial (RCT). This three-arm trial, randomised by region and district, assessed the introduction of $\mathrm{PB}$ in rural settlements in Russia. The trial compared introduction of $\mathrm{PB}$ assisted by administrative training, to training plus additional technical assistance from two fulltime consultants, and included control districts that received neither training nor consultancy assistance [21].

There were thirteen quantitative observational studies. Nine studies analysed population level data using linear regression techniques [8, 9, 22-28]; three studies used regression models to analyse individual survey data [29-32], and one study used cross tabulation descriptive analysis to assess population level data [33].

\section{Case studies}

Twenty-three evaluations were case studies providing descriptive analyses of single $(n=10)$ or multiple $(n=13)$ instances of PB. The methods used for these case studies included participant observation $(n=9)$ [34-38], interviews $(n=15)[18,34-46]$, surveys of PB participants and PB officials $(n=14)[18,19,34-37,39,42,46-51]$, or descriptive analysis of municipal data $(n=6)[17,19,20,40,41,43]$, fifteen studies applied various combinations of these methods.

What outcomes have been used to investigate the effects of PB processes?

We define three broad categories of outcomes: political, economic and budgetary, and population outcomes (Table 3). The outcomes for each category are described below. While assessing the methodological quality of the studies was beyond the scope of this review, we include an indication of the results reported for interest (Additional file 1: Table S3).

\section{Political outcomes}

Outcomes we labelled as 'political' were reported by two thirds $(n=26)$ of studies. These outcomes included: participation in PB by disadvantaged sections of the population; impacts on further involvement in political or civic activities; and learning about political processes as a result of engagement in PB. The majority $(n=20)$ of political outcomes were evaluated by descriptive case studies. Thirteen studies examined participation in PB of disadvantaged groups including women, people with low income, low education, disability, or of an ethnic minority, with overall mixed results reported. Several observational studies reported some increase in participation $[8,21,22,29,32]$. The case studies reported more equivocal findings, levels of participation by disadvantaged groups was increased [37], low [20, 47] or mixed [41]; barriers remained [43] and active participation (i.e. taking part in discussions) was found only to increase with increased years of involvement in PB $[38,50]$.

The relationship between $\mathrm{PB}$ and further involvement in political or civic activities was explored in six studies $[30,31,34,35,37,42,51]$, sometimes reporting that the individuals engaging in $\mathrm{PB}$ were already involved in other civil society organisations. Changes in how people acted in political arenas as a result of $\mathrm{PB}$ were examined by nine studies $[18,30,32,36,39,42,44,46,48]$, tending to report positive impacts. Learning about political processes as a result of engagement in $\mathrm{PB}$ was examined by four case studies $[19,37,42,52]$, in general reporting some increased learning of political processes.

\section{Economic and budgetary outcomes}

Twelve studies reported on economic or budgetary outcomes such as local tax revenue or local government spending on public services such as healthcare, sanitation and housing. Two studies analysed impacts on local tax revenue from municipal records [21, 24], using fixed effect regression analysis to examine whether there was a change in local tax revenue according to the implementation of $\mathrm{PB}$, reporting positive results.

Thirteen studies examined the allocation of PB funds. Three studies set in Brazil assessed spending on healthcare and sanitation, comparing municipalities that implemented PB with comparable areas that did not, reporting positive results $[9,25,26]$. All three studies applied linear regression analysis to large datasets: for all Brazilian municipalities with available data [25]; and for municipalities in Brazil with a population of over 100,000 inhabitants, 220 municipalities from 1991 to 2000 [9] and 253 municipalities between 1989 and 2008 
Table 3 Cross-tabulation of study design by outcome category

\begin{tabular}{|c|c|c|c|c|}
\hline Outcomes & Case studies single & $\begin{array}{l}\text { Case studies } \\
\text { multiple }\end{array}$ & $\begin{array}{l}\text { Quantitative Observational Study } \\
\text { (Population level data) }\end{array}$ & RCT \\
\hline $\begin{array}{l}\text { Population Social } \\
\text { Impacts (poverty, health, education, housing, } \\
\text { sanitation) }\end{array}$ & $\begin{array}{l}\text { Kendall } 2015[18] \\
\text { Walker 2013/2016 } \\
{[44,45]}\end{array}$ & & $\begin{array}{l}\text { a'Biderman } 2007 \text { [24] } \\
\text { a'Boulding } 2010 \text { [9] } \\
\text { a'Goncalves } 2014 \text { [25] } \\
\text { a'Touchton } 2014[26] \\
\text { a'WorldBank } 2008[8]\end{array}$ & \\
\hline $\begin{array}{l}\text { Economic and budgetary (funding of public } \\
\text { services) }\end{array}$ & Stewart 2014 [20] & $\begin{array}{l}\text { Cabannes } 2005 \\
\text { [49] } \\
\text { Cabannes } 2015 \\
\text { [39] } \\
\text { Del Prado } 2015 \\
\text { [40] } \\
\text { Dzinic } 2016 \text { [17] }\end{array}$ & $\begin{array}{l}\text { Biderman } 2007 \text { [24] } \\
\text { Boulding } 2010 \text { [9] } \\
\text { Da Silva } 2014 \text { [33] } \\
\text { Goncalves } 2014 \text { [25] } \\
\text { Jaramillo } 2013 \text { [23] } \\
\text { Jaramillo } 2015 \text { [27] } \\
\text { Touchton } 2014 \text { [26] } \\
\text { a'WorldBank } 2008 \text { [8] } \\
\text { Grillos } 2017 \text { [28] }\end{array}$ & $\begin{array}{l}\text { Beuermann } \\
2014[21]\end{array}$ \\
\hline $\begin{array}{l}\text { Political (participation, democratic processes, } \\
\text { political systems) }\end{array}$ & $\begin{array}{l}\text { Abers } 1998 \text { [34] } \\
\text { Baiocchi } 2001 \text { [47] } \\
\text { Baiocchi } 2003[35] \\
\text { Célérier } 2015[36] \\
\text { Hernandez } 2010 \\
\text { [41] } \\
\text { a'Kendall } 2015 \text { [18] } \\
\text { aStewart 2014 [20] } \\
\text { a'Walker 2013/2016 } \\
\text { [44, 45] } \\
\text { Hajdarowicz } 2018 \\
\text { [63] }\end{array}$ & $\begin{array}{l}\text { Bassoli } 2012 \text { [48] } \\
\text { a'Cabannes } 2015 \\
\text { [39] } \\
\text { Luchmann } 2012 \\
\text { [42] } \\
\text { McNulty } 2015 \text { [43] } \\
\text { Montambeault } \\
2016 \text { [50] } \\
\text { Nylen } 2003 \text { [37] } \\
\text { Renno } 2010 \text { [51] } \\
\text { SQW Consulting } \\
\text { 2011 [19] } \\
\text { Talpin 2012 [38] } \\
\text { Wu 2011 [46] } \\
\text { Gregorčič 2016 } \\
\text { [52] }\end{array}$ & $\begin{array}{l}\text { Borba } 2012[32] \\
\text { Garcia } 2011[29] \\
\text { Schneider } 2002[22] \\
\text { Wampler } 2007 / 2012^{\mathrm{b}}[30,31] \\
\text { a'WorldBank } 2008[8]\end{array}$ & \\
\hline
\end{tabular}

${ }^{\text {a }}$ Study has outcomes in more than one outcome category

b Wampler 2007/2012 analysis of survey data. Multiple dates indicates same data used in two articles

[26]. Analysis of PB in Indonesia found areas with more low income households were less likely to benefit from the PB process [28]. Linear regression was also used to examine allocation of $\mathrm{PB}$ funding in relation to citizens' priorities in Russian settlements [21]. The impact of $\mathrm{PB}$ on the number and quality of agricultural services provided in 100 randomly selected Peruvian municipalities was assessed using linear regression analysis of data from the municipalities and central government [27]. The impact of $\mathrm{PB}$ on the provision of water services in 219 districts in Peru was analysed using linear regression, reporting no improvement to the services [23]. Budgetary expenditure on public services including water and sanitation in Porto Alegre in Brazil was analysed using difference in difference regression analysis, reporting positive results [8]. Municipal budgetary and census data from the Brazilian Institute of Geography and Statistics was used to conduct a descriptive analyse of the allocation of $\mathrm{PB}$ funds according to level of deprivation, finding mixed results [33]. Four further studies examined which public services received funding from $\mathrm{PB}$, which included provision of recreational public spaces $[17,20,49]$, and micro credit centres [40].

\section{Health and social outcomes}

Health or social outcomes, such as infant mortality, poverty rates, education, and access to sanitation, were evaluated by eight studies, most studies examining multiple outcomes. The impact of $\mathrm{PB}$ on infant mortality was examined by five quantitative observational studies located in Brazil $[8,9,24-26]$. These studies report mixed results. The two more recent studies reported that $\mathrm{PB}$ reduced infant mortality $[25,26]$. Poverty rates in municipalities implementing PB were assessed by two studies in Brazil, both of which reported reductions in poverty $[8,9]$. Education, measured as either child or adult literacy or years of school attendance, was investigated by two quantitative observational studies and one case study, with mixed results $[9,18,24]$. Access to sanitation, piped water supply and sewerage, was measured by one observational study and one case study, reporting positive results $[8,39]$.

\section{Discussion}

This scoping review provides a systematic analysis of studies evaluating health and wellbeing impacts of PB. We identified 37 evaluation studies focussing on issues relating to health and wellbeing or delivery of public 
services, a relatively small number in comparison with the estimated 2000 to 2700 PB schemes implemented worldwide [11, 53]. While a few evaluations used population level datasets to examine health outcomes in South America, overall there was limited scope in the methods used to assess $\mathrm{PB}$, and the vast majority of studies focused on political outcomes.

The majority of studies found were single or multiple case studies, describing the scenario of individual PB schemes. There were fewer observational quantitative modelling studies using large population datasets, and only one study that combined this with qualitative analysis. This dearth of mixed methods approaches is somewhat puzzling. PB interventions are intended to realise a complex range of democratic and social goods through both the processes and outcomes of public participation in budgetary decision-making. Mixed methods approaches, with their capacity to combine exploratory and explanatory research designs $[54,55]$, are uniquely suited to conduct multidimensional evaluations of both processes and outcomes [56]. While further detail of mixed methods may be included in book length descriptions of $\mathrm{PB}$ processes (e.g. [57-59]), our review suggests that there is considerable scope for methodological development and innovation using mixed methods approaches to evaluate PB.

The majority of studies identified in this review were evaluations of PB in South America. All of the quantitative observational studies analysing population level data were conducted in South America, with the exception of the randomised controlled trial conducted in Russia. Evaluation of PB in Brazil is possible due to the availability of population data aggregated at municipal level. Such data is not routinely available in many countries [60]. Robust evaluation of PB in other countries may require developing databases at local government level. The concentration of $\mathrm{PB}$ evaluation in Brazil has strong implications for policy makers in countries beyond South America interested in implementing PB. As PB has been developed in other continents, and in high income countries, the processes, budgets, and scale of the initiatives have become more varied [13, 61]. Also, the rationale of social justice underpinning $\mathrm{PB}$ when it began in Porto Alegre has often become marginalised in $\mathrm{PB}$ outside Brazil [13]. While the results of PB in Brazil may be generalizable to some other countries with similar levels of existing public services, in general, these differences suggest that the results of evaluations on health and wellbeing outcomes found in PB in Brazil may not translate to $\mathrm{PB}$ in other contexts. The main focus of $\mathrm{PB}$ evaluations was on political outcomes, we found less evaluation of outcomes relating to delivery of public services or assessing health outcomes or wellbeing outcomes related to poverty. In part, this may relate to who is conducting the analysis; as far as we could determine, the majority of studies were conducted within the fields of political science and public administration. Despite worldwide implementation of $\mathrm{PB}$, this review finds that the implications for health and wellbeing have not been the focus of attention in public health literature. In our review, the exception is provided by Boulding and Wampler [9], who discuss the possible impacts on wellbeing, finding limited research in this area. More recently, studies have accessed population level datasets to investigate health impacts, focussing on outcomes such as infant mortality, education and poverty rates (e.g. $[25,26])$. However, the limited evaluations of PB health and wellbeing outcomes may also relate to the ad hoc quality of many PB processes. Only Brazil has institutionalised PB to the point where comparative and longitudinal evaluations become viable, which accentuates the challenge of assessing health and wellbeing outcomes. Nevertheless, recent policy developments in other countries (e.g. Harkins et al. $[12,13,62])$ are seeking to embed PB in local government for the long term. This offers the opportunity for a PB evaluation agenda that transcends the geographic and thematic foci prevalent in the field.

\section{Strengths and limitations of this review}

We used systematic, transparent methods with predetermined inclusion criteria. It is possible that some articles may not have been identified in the literature search, however, we are confident that the search of twenty-one databases provided us with a broad sweep of international peer-reviewed articles and grey literature on PB. As this was a scoping review, we did not make a formal assessment of the risk of bias in the included studies. Our aim was to map out what evaluations relating to health and wellbeing have been conducted. This section outlines key learning points and implications, in particular regarding the methodological, geographic, and thematic foci that dominate the field of $\mathrm{PB}$ evaluations.

\section{Conclusion}

The findings of this review lead us to recommend further evaluation of the impact of $\mathrm{PB}$ on health and wellbeing be conducted in a range of national contexts. The lack of substantial evaluations outside Brazil, and the variations of $\mathrm{PB}$ being implemented worldwide, support the recommendation that when $\mathrm{PB}$ is being adopted, adapted and initiated, this should be accompanied by rigorous evaluation of the process and expected outcomes, using appropriate comparators. Governments involved in supporting and developing $\mathrm{PB}$ processes are uniquely placed to ensure that evaluation is not an afterthought, but an embedded component of robust PB policy over the long term. PB has spread globally, partly on the basis of claims regarding its potential to empower 
communities and improve people's lives. Our review indicates that the PB field needs a stronger evidence base in order to substantiate and refine those claims in a variety of contexts.

\section{Additional file}

Additional file 1: Tables S1a and S1b Databases searched. Table S2 Data extraction template. Table S3 Detailed characteristics of studies. (DOCX $30 \mathrm{~kb})$

\section{Abbreviations}

PB: Participatory budgeting; UK: United Kingdom

\section{Acknowledgements}

We would like to thank Valerie Wells, Information Scientist, for conducting the updated literature searches. We are also thankful for the useful advice provided by the peer reviewers.

\section{Funding}

The study was funded by What Works Scotland (ESRC ES/M003922/1 and Scottish Government). ESRC and Scottish Government had no role in the design of this study, in the collection, analysis, and interpretation of the data or in writing the manuscript. MC and PC are supported by the Medical Research Council and the Scottish Government Chief Scientist Office (MC_UU_12017-15 and SPHSU15).

\section{Availability of data and materials}

All data generated or analysed during this study are included in this published article and its Additional files.

\section{Authors' contributions}

PC and $\mathrm{OE}$ conceived the idea of the study. $\mathrm{MC}$ and $\mathrm{PC}$ designed the review methodology. CF conducted the literature search. MC, PC and OE conducted screening of articles and data extraction. MC wrote the first draft of the manuscript; all authors critically reviewed subsequent drafts. All authors have approved the final version of the manuscript.

\section{Competing interest}

The authors declare that they have no competing interests.

\section{Ethics approval and consent to participate}

Not applicable.

\section{Consent for publication}

Not applicable.

\section{Publisher's Note}

Springer Nature remains neutral with regard to jurisdictional claims in published maps and institutional affiliations.

\section{Author details}

'MRC/CSO Social and Public Health Sciences Unit, University of Glasgow, 200 Renfield Street, Glasgow G2 3QB, UK. ²What Works Scotland, Glasgow, UK.

${ }^{3}$ University of Edinburgh, Edinburgh, UK.

Received: 25 January 2018 Accepted: 21 June 2018

Published online: 03 July 2018

\section{References}

1. Dias N. Hope for democracy: 25 years of participatory budgeting worldwide. Sao Bras de Alportel: In Loco Association; 2014

2. Wampler B. A Guide to participatory budgeting. In: Shah A, editor. Participatory budgeting. Washington D.C: World Bank; 2007

3. Shah A, editor. Participatory budgeting. Washington, DC: World Bank Publications; 2007

4. Sintomer Y, Herzberg C, Röcke A, Allegretti G. Modelos transnacionais de participação cidadã: o Caso do orçamento participativo [transnational models of citizen participation: the case of participatory budgeting]. J Public Deliberation. 2012;8(2):9.

5. Heimans J. Strengthening participation in public expenditure management. OECD development Centre policy brief 22. Paris: organisation for economic cooperation and. Development. 2002;

6. Cabannes Y. Participatory budgeting: conceptual framework and analysis of its contribution to urban governance and the millenium development goals. Quito: UN-HABITAT; 2004.

7. Cabannes Y. Contribution of participatory budgeting to provision and management of basic services: Municipal practices and evidence from the field. London: IIED working paper; 2014.

8. World Bank. Brazil: toward a more inclusive and effective participatory budget in Porto Alegre, volume 1 main report. Washington DC: The World Bank; 2008.

9. Boulding C, Wampler B. Voice, votes, and resources: evaluating the effect of participatory democracy on well-being. World Dev. 2010;38(1):125-35.

10. Cabannes Y. 72 frequently asked questions about participatory budgeting Nairobi: UN-HABITAT; 2004

11. Baiocchi G, Ganuza E. Popular democracy: the paradox of participation. Stanford: Stanford University Press; 2017.

12. Harkins C, Moore K, Escobar O. Review of 1st generation participatory budgeting in Scotland. Edinburgh: What Works Scotland; 2016.

13. Ganuza E, Baiocchi $G$. The power of ambiguity: how participatory budgeting travels the globe. J Public Deliberation. 2012;8(2):1-12.

14. Levac D, Colquhoun H, O'Brien KK. Scoping studies: advancing the methodology. Implement Sci. 2010; https://doi.org/10.1186/1748-5908-5-69.

15. Arksey H, O'Malley L. Scoping studies: towards a methodological framework Int J Soc Res Methodol. 2005:8(1):19-32.

16. Campbell M, Craig P, Escobar O. The impact of Participatory Budgeting: a scoping review of evaluations and outcomes. Review protocol. Glasgow: University of Glasgow; 2016. (https://www.gla.ac.uk/researchinstitutes/ healthwellbeing/research/mrccsosocialandpublichealthsciencesunit/ sharingourevidence/reportsandprotocols/)

17. Džinic J, Murray SM, Ewa M-B. Participatory budgeting: a comparative study of Croatia, Poland and Slovakia. NISPAcee J Public Administration and Policy. 2016:9(1):31-56

18. Kendall N, Kaunda Z, Friedson-Rideneur S. Community participation in international development education quality improvement efforts: current paradoxes and opportunities. Educ Assess Eval Acc. 2015;27(1):65-83.

19. SQW Consulting. Communities in the driving seat: a study of participatory budgeting in England. Final report. London: Department for Communities and Local Government; 2011.

20. Stewart LM, Miller SA, Hildreth RW, Wright-Phillips MV. Participatory budgeting in the United States: a preliminary analysis of Chicago's 49th Ward experiment. New Political Science. 2014:36(2):193-218.

21. Beuermann D, Amelina M. Does participatory budgeting improve decentralized public service delivery? Inter-American Development Bank; 2014

22. Schneider A, Goldfrank B. Budgets and ballots in Brazil. Participatory budgeting from the city to the state. Brighton: Institute of Development Studies: 2002. Report no. 1858644062

23. Jaramillo M, Alcázar L. Does participatory budgeting have an effect on the quality of public services? The case of Peru's water and sanitation sector. Washington DC: Inter-American Development Bank; 2013.

24. Biderman C, da Silva GP. Estimating the impact of participatory budget on observed outcomes. Associação Nacional dos Centros de Pósgraduação em Economia (ANPEC) [Brazilian Association of Graduate Programs in Economics]; 2007.

25. Goncalves $\mathrm{S}$. The effects of participatory budgeting on municipal expenditures and infant mortality in Brazil. World Dev. 2014:53:94-110.

26. Touchton M, Wampler B. Improving social well-being through new democratic institutions. Comp Polit Stud. 2014:47(10):1442-69.

27. Jaramillo M, Wright GD. Participatory democracy and effective policy: is there a link? Evidence from rural Peru. World Dev. 2015:66:280-92.

28. Grillos T. Participatory budgeting and the poor: tracing bias in a multistaged process in solo, Indonesia. World Dev. 2017:96:343.

29. Garcia MJR, Mora CM, Yanez CJN. Performance of participation mechanisms: gender and institutional design. Territorial councils and participatory budgets in Spanish cities. Rev CLAD Reforma Democr. 2011;50:143.

30. Wampler B. Can participatory institutions promote pluralism? Mobilizing low-income citizens in Brazil. Stud Comp Int Dev. 2007;41(4):57-78. 
31. Wampler B. Entering the state: civil society activism and participatory governance in Brazil. Polit Stud. 2012;60(2):341-62.

32. Borba J, Ribeiro EA. Orçamento Participativo e cultura política: Explorando as relações entre inovação institucional, valores e atitudes políticas. [Participatory budgeting and political culture: exploring the relation between institutional innovation and political values and attitudes.]. Politica \& Sociedade: Revista de Sociologia Politica. 2012;11(21):13-44.

33. da Silva R. Desenho institucional e promoção da justiça em espaços participativos: implicações em um estudo de caso [Institutional design and promotion of justice in participatory spaces: implications in a case study]. Opiniao Publica. 2014;20(2):252-72.

34. Abers R. From clientelism to cooperation: local government, participatory policy, and civic organizing in Porto Alegre, Brazil. Polit Soc. 1998;26(4):511-37.

35. Baiocchi G. Emergent public spheres: talking politics in participatory governance. Am Sociol Rev. 2003;68(1):52-74.

36. Célérier L, Cuenca Botey LE. Participatory budgeting at a community level in Porto Alegre: a Bourdieusian interpretation. Account Audit Account J. 2015; 28(5):739-72.

37. Nylen WR. Examining the claims of proponents of the participatory budget. Basingstoke: Palgrave; 2003.

38. Talpin J. What can ethnography bring to the study of deliberative democracy? Evidence from a study on the impact of participation on actors. Rev Int Sociol. 2012;70(2):143-63.

39. Cabannes $Y$. The impact of participatory budgeting on basic services: municipal practices and evidence from the field. Environ Urban. 2015; 27(1):257-84

40. del Prado F, Rosellon MAD, Florendo GA. Grassroots participatory budgeting process in Negros Province. Makati City: Philippine Institute for Development Studies; 2015.

41. Hernandez-Medina E. Social inclusion through participation: the case of the participatory budget in Sao Paulo. Int J Urban Region. 2010;34(3):512-32.

42. Luchmann LHH. Participação e aprendizado político no orçamento participativo: estudo de caso em um município catarinense [Political participation and learning in participative budget: a case study in a commune of Santa Catarina, Brazil]. Educ Soc. 2012:33(119):513-32.

43. McNulty SL. Barriers to participation: exploring gender in Peru's participatory budget process. J Dev Stud. 2015:51(11):1429.

44. Walker APP. Embodied identity and political participation: squatters' engagement in the participatory budget in Brazil. Ethos. 2013;41(2):199-222.

45. Walker APP. Self-help or public housing? Lessons from co-managed slum upgrading via participatory budget. Habitat Int. 2016;55:58-66.

46. Wu Y, Wang $W$. The rationalization of public budgeting in China: a reflection on participatory budgeting in Wuxi. Public Financ Manag. 2011; 11(3):262-83.

47. Baiocchi G. Participation, activism, and politics: the Porto Alegre experiment and deliberative democratic theory. Polit Soc. 2001;29(1):43-72.

48. Bassoli M. Participatory budgeting in Italy: an analysis of (almost democratic) participatory governance arrangements. Int J Urban Region. 2012;36(6): 1183-203.

49. Cabannes Y. Children and Young people build participatory democracy in Latin American cities. Child Youth Environ. 2005;15(2)185-210.

50. Montambeault F. Participatory citizenship in the making? The multiple citizenship trajectories of participatory budgeting participants in Brazil. J Civil Soc. 2016:12(3):282-98

51. Renno LR, Ames B. Participatory budgeting, political discussion networks and political information in two Brazilian cities. In: Political discussion in modern democracies: a comparative perspective. London: Routledge Taylor \& Francis Group; 2010. p. 62-78.

52. Gregorčič M, Krašovec SJ. Social and learning practices in participatory democracy process the case study of self-organized communities in Maribor, Slovenia, contextualised through the e-participatory budgeting in Reykjavík. Iceland Sodob Pedagog. 2016;67(4):168-82.

53. Sintomer $Y$, Herberg C, Allegretti $G$, Rocke A, Alves M. Learning from the south: participatory budgeting worldwide updated version-An Invitation to global cooperation. Dialog Global 25. Bonn, GDR: Capacity Building International, Germany/Service Agency Communities in One World; 2013.

54. Creswell JW, Plano Clark V. Designing and conducting mixed methods research. London: SAGE; 2011.

55. Manfred Max B, editor. Advances in mixed methods research: theories and applications. London: SAGE; 2008.
56. Greene JC, Caracelli VJ, Graham WF. Toward a conceptual framework for mixed-method evaluation designs. Educ Eval Pol An. 1989:11(3):255-74.

57. Wampler B. Participatory budgeting in Brazil: contestation, cooperation, and accountability. University Park: Pennsylvania State University Press; 2007.

58. Baiocchi G, Heller P, Silva M. Bootstrapping democracy: transforming local governance and civil Society in Brazil. Stanford: Stanford University Press; 2011.

59. Goldfrank B. Deepening local democracy in Latin America : participation, decentralization, and the left. University Park: Pennsylvania State University Press; 2011.

60. Vlahov D, Caiaffa WT. Healthy urban governance and population health: participatory budgeting in Belo Horizonte, Brazil. In: The urban transformation: health, shelter and climate change: Taylor and Francis: 2013. p. 63-81.

61. Sintomer $Y$, Herzberg C, Röcke A. Participatory budgeting in Europe: potentials and challenges. Int J Urban and Region. 2008;32(1):164-78.

62. Escobar O, Garven A, Harkins C, Glazik K, Cameron S, Stoddart A. Participatory budgeting in Scotland: The interplay of public service reform, community empowerment and social justice. In: Dias N, editor. Hope for Democracy: 30 years of participatory budgeting worldwide. Sao Bras de Alportel: In Loco Association; 2018.

63. Hajdarowicz I. Does participation empower? The example of women involved in participatory budgeting in Medellin. J Urban Aff. 2018: https:// doi.org/10.1080/07352166.2018.1431048

\section{Ready to submit your research? Choose BMC and benefit from:}

- fast, convenient online submission

- thorough peer review by experienced researchers in your field

- rapid publication on acceptance

- support for research data, including large and complex data types

- gold Open Access which fosters wider collaboration and increased citations

- maximum visibility for your research: over $100 \mathrm{M}$ website views per year

At BMC, research is always in progress.

Learn more biomedcentral.com/submissions 